\title{
Mirage simulations of the massiv sample
}

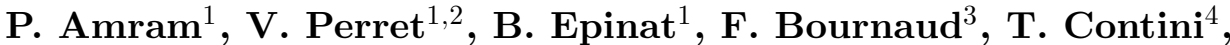 C. Divoy ${ }^{4}$, B. Garilli ${ }^{5}$, M. Kissler-Patig ${ }^{6}$, O. Le Fevre ${ }^{1}$, C. Lopez-Sanjuan ${ }^{7}$, J. Moultaka ${ }^{4}$, L. Pairo ${ }^{5}$, E. Perez-Montero ${ }^{8}$, J. Queyrel ${ }^{4}$, L. Tasca ${ }^{1}$, L. Tresse ${ }^{1}$ and D. Vergani ${ }^{9}$}

${ }^{1}$ LAM, Marseille (F), ${ }^{2} \mathrm{U}$. Zurich, (Ch), ${ }^{3} \mathrm{CEA}$, SAp, AIM, Saclay $(\mathrm{F}),{ }^{4}$ IRAP, Toulouse (F), ${ }^{5}$ INAF-IASFBO, Bologna (I), ${ }^{6}$ GEMINI, Hilo (USA), ${ }^{7}$ FEFCA, Teruel(E), ${ }^{8}$ IAC, Granada(E),

${ }^{9}$ INAF-IASF, Milano (I). Email: philippe.amram@lam.fr

The MIRAGE sample (Merging \& isolated high-redshift AMR galaxies; Perret 2014, PhD dissertation; Perret et al. 2014, AA 562, 1) has been built in order to understand the contribution of the merger processes to the mass assembly in the MASSIV (Mass Assembly Survey with SINFONI in VVDS, Contini et al. 2012, AA 539, 91) sample. It consists of a sample of idealized simulations based on the RAMSES code; the initial conditions were designed to reproduce the physical properties of the most gas-rich young galaxies. The sample is composed of 20 simulations of mergers exploring the initial parameters of mass and orientation of the disks with a spatial resolution reaching 7 parsecs.

The MIRAGE simulations show $(i)$ an absence of star formation bursts in mergers of fragmented and turbulent disks, suggesting a saturation mechanism; $(i i)$ that the gas rich clump merging mechanism is able to control the bulge mass growth, to erode the central profile of the dark matter halo and to drive massive gas outflows into the disk plane; (iii) whatever the orbital configuration (prograde or retrograde) and whatever the mass ratio between the disks $(1 / 3 / 6-\log M=10.6 / 10.2 / 9.8)$, a new disk of gas is reconstructed quickly after the merger. This is due to the high gas fraction at the merging event $(60 \%)$.

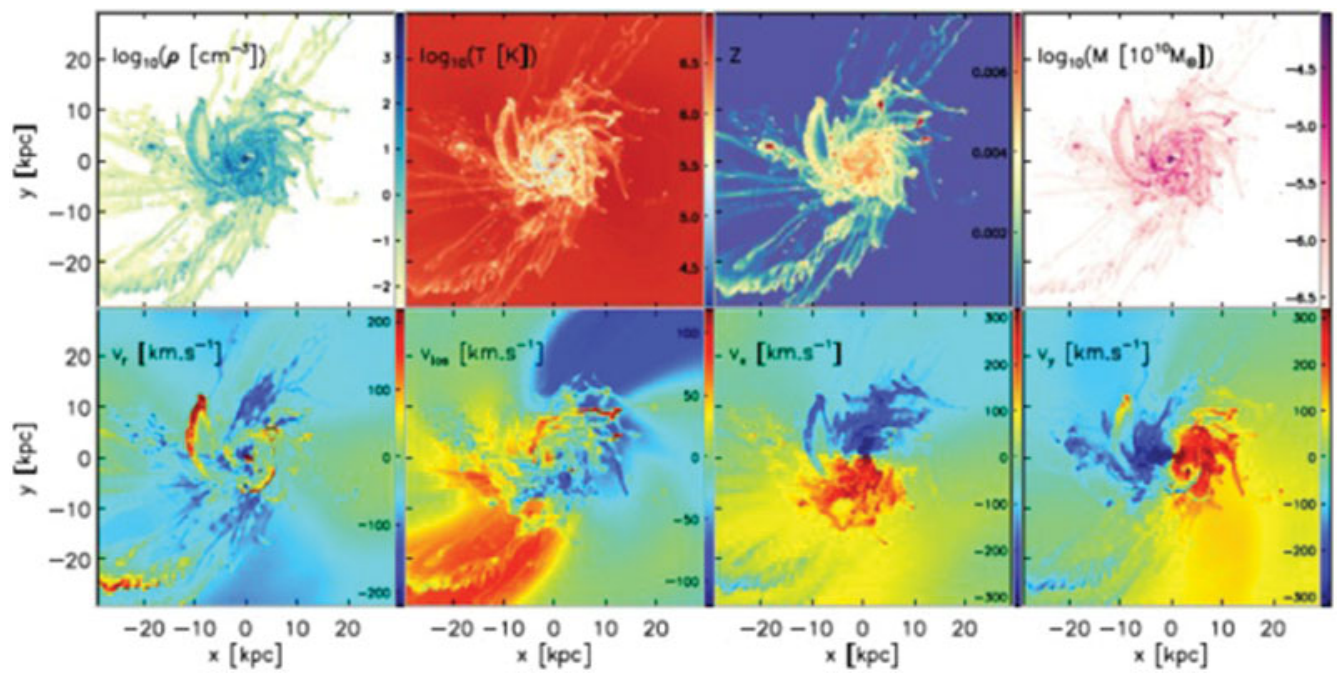

Figure 1. We carry out a comparative study of the MASSIV kinematical data to a set of more than 4000 pseudo-observations at $\mathrm{z}=1.7$ built from simulations of the MIRAGE sample to determine the ability to detect galaxy merger signatures under the observational conditions of the SINFONI instrument. Upper panels from left to right: density, temperature, metallicity and mass maps. Bottom panels from left to right: radial cylindric velocity in the plane of the disk, line-of-sight velocity and the two last ones are projections on the two planes perpendicular to the sky plane (Perret 2014, $\mathrm{PhD}$ dissertation). 